Article

\title{
Design, Synthesis, and Biological Evaluation of a New Series of Biphenyl/Bibenzyl Derivatives Functioning as Dual Inhibitors of Acetylcholinesterase and Butyrylcholinesterase
}

\author{
Dong-mei Wang ${ }^{1}$, Bo Feng ${ }^{1}$, Hui Fu ${ }^{2}$, Ai-lin Liu ${ }^{1}$, Lin Wang ${ }^{1}$, Guan-hua Du ${ }^{1}$ and Song Wu ${ }^{1, *}$ \\ 1 State Key Laboratory of Bioactive Substances and Functions of Natural Medicines \& Ministry of Health Key \\ Laboratory of Biosynthesis of Natural Products, Institute of Materia Medica, Chinese Academy of Medical \\ Sciences \& Peking Union Medical College, Beijing 100050, China; wangdmchina@imm.ac.cn (D.-m.W.); \\ fengbo@imm.ac.cn (B.F.); liuailin@imm.ac.cn (A.-l.L.); wlin@imm.ac.cn (L.W.); dugh@imm.ac.cn (G.-h.D.) \\ 2 Beijing Institutes For Drug Control, Beijing 100050, China; Fuhuifree@sina.com \\ * Correspondence: ws@imm.ac.cn; Tel.: +86-10-8316-3542
}

Academic Editors: Diego Muñoz-Torrero and Derek J. McPhee

Received: 19 October 2016; Accepted: 16 January 2017; Published: 20 January 2017

\begin{abstract}
Alzheimer's disease (AD), the most common form of dementia in adults, is a progressive neurodegenerative disorder of the brain characterized by loss of memory and steady deterioration of cognition. Here, a series of symmetrical molecules containing biphenyl/bibenzyl scaffolds (12-36) were designed, synthesized, and evaluated for their ability to inhibit both acetylcholinesterase (AChE) and butyrylcholinesterase (BuChE). A biological evaluation showed that most of these biphenyl derivatives were potent $\mathrm{AChE}$ and $\mathrm{BuChE}$ inhibitors. Among them, compound 15 displayed the greatest ability to inhibit $\mathrm{BuChE}\left(\mathrm{IC}_{50}=0.74 \mu \mathrm{M}\right)$ and was also a good $\mathrm{AChE}$ inhibitor $\left(\mathrm{IC}_{50}=1.18\right.$ $\mu \mathrm{M})$. Compound 19 was not only a potent $\mathrm{AChE}$ inhibitor $\left(\mathrm{IC}_{50}=0.096 \mu \mathrm{M}\right)$, but also a mild BuChE inhibitor $\left(\mathrm{IC}_{50}=1.25 \mu \mathrm{M}\right)$. Overall, these results suggested that compound 19 may be a promising agent in the treatment of $\mathrm{AD}$.
\end{abstract}

Keywords: Alzheimer's disease; biphenyl/bibenzyl derivatives; acetylcholinesterase inhibitors; butyrylcholinesterase inhibitors; molecular docking

\section{Introduction}

Alzheimer's disease (AD), the most common form of dementia in adults, is a progressive neurodegenerative disorder of the brain characterized by loss of memory and steady deterioration of cognition. This disease currently affects more than 30 million people worldwide [1]. However, the exact pathophysiology of AD remains unclear. Several findings indicate that amyloid- $\beta$ plaques [2], tau protein aggregation [3], oxidative stress [4,5], and a low level of acetylcholine in the brain play important roles in the pathophysiology of the disease [6]. At present, no drug is available to decrease, reverse, or stop the pathological process of AD. Although several research strategies have been envisaged recently, the current therapeutic option is limited to only four AChE inhibitors [7,8], namely, donepezil, rivastigmine, galantamine, and tacrine (now discontinued), and one $N$-methyl-D-aspartate receptor antagonist, memantine [9].

However, two types of cholinesterase (ChE) enzymes have been found in the central nervous system, AChE and BuChE. Studies have indicated that BuChE catalyzes the hydrolysis of acetylcholine in the brains of normal people, as well as patients with Alzheimer's disease (AD) [10]. While ACh activity decreases to $10 \%-15 \%$ of normal values in certain brain regions in patients with mild to severe $\mathrm{AD}, \mathrm{BuChE}$ levels remain unchanged, or even increase, with disease progression [11]. Therefore, mixed 
AChE/BuChE inhibitors may result in higher efficacy for treatment of AD. Rivastigmine, used in AD treatment, is a potent inhibitor of both enzymes [12]. Selective BuChE inhibitors cause an elevation of acetylcholine, augment long-term potentiation, and enhance learning. They have also been shown to decrease amyloid- $\beta$ in a rodent model of AD [13]. Therefore, dual AChE/BuChE inhibitors may have better clinical efficacy without remarkable adverse effects.

To find new ChE inhibitors, structure-based virtual screening and high throughput screening were used in the present study to screen the Institute of Meteria Medica's library of compounds, which contains more than 10,000 molecules. A small symmetrical molecule, 4,4'-bi-( $\beta$-dimethylamino-propinoyl)-biphenyl (1, Figure 1), was discovered to be a novel dual inhibitor of $\mathrm{AChE}$ and $\mathrm{BuChE}$, with a concentration required for $50 \%$ inhibition of the maximal response in vitro (its $\mathrm{IC}_{50}$ value) of $0.75 \mu \mathrm{M}$ for $\mathrm{AChE}$ and $0.19 \mu \mathrm{M}$ for BuChE.

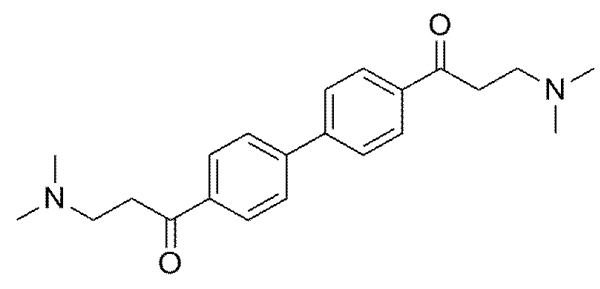

1

Figure 1. Chemical Structure of $\mathbf{1}$.

The biphenyl scaffold is a privileged structure [14] and it exists in $4.3 \%$ of the molecules in commercially available drugs [15]. However, only limited studies have examined these types of compounds for their anti-AChE and anti-BuChE activities. Thus, to find potential anti-AD agents, we report here on the synthesis of a series of derivatives and analogues of compound 1 . The chemical modifications included the following: (i) replacing the dimethylamino group with appropriate secondary amines and heterocyclic amines; (ii) changing the length of the linkers that connect the biphenyl carbonyl core with an amine moiety; (iii) alternating the rigid biphenyl core with a flexible bibenzyl; and (iv) reducing the carbonyl group to a hydroxyl group.

\section{Results and Discussion}

\subsection{Chemistry}

The synthetic route for these target compounds (12-36) is outlined in Scheme 1. Commercially available biphenyl 2 or bibenzyl 3 reacted with $\mathrm{Cl}\left(\mathrm{CH}_{2}\right)_{n} \mathrm{COCl}(n=1-4)$ in the presence of $\mathrm{AlCl}_{3}$ in dry $\mathrm{CS}_{2}$ at $40-50{ }^{\circ} \mathrm{C}$ to generate intermediates (4-11) with yields of $71 \%-82 \%$. The target compounds (12-33) were synthesized by substitution of chlorine in the intermediates (4-11) with appropriate secondary alkyl amines or heterocyclic amines. The reaction was carried out in $\mathrm{CH}_{3} \mathrm{CN}$ with $\mathrm{K}_{2} \mathrm{CO}_{3}$ and $\mathrm{KI}$. The yields were $52 \%-65 \%$. Reduction of compounds 19, 20, and 30 with sodium borohydride in THF gave the desired alcohols (34-36) with yields of 52\%-57\%. Diketones $(\mathbf{1 9}, \mathbf{2 0}$, and $\mathbf{3 0})$ are symmetrical compounds, but two asymmetrical carbon centers can be produced during the reduction process to generate compounds 34-36. Thus, the final products may have existed as three isomers, namely, distereomeric mixtures of a meso form $(R, S / S, R)$, and a racemic mixture of two chiral compounds $(R, R)$ and $(S, S)$. These isomers can be separated by chiral column chromatography. However, as our aim is only to investigate whether the carbonyl groups in the molecules were crucial for biological activities, the separation was not conducted. Twenty-one compounds were novel, and the other four compounds have been previously reported [16], although neither the synthetic route nor the structure-activity relationships were provided. The crude products were purified in the present study by column chromatography on silica gel eluted with $\mathrm{CH}_{2} \mathrm{Cl}_{2}$ and $\mathrm{MeOH}$. The purities of all compounds were higher than $99 \%$. The structures of the target compounds are shown in Table 1. 
Table 1. AChE and BuChE inhibition by target compounds 12-36.

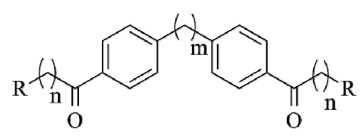

$12 \sim 33$

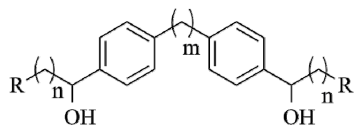

$34 \sim 36$

\begin{tabular}{|c|c|c|c|c|c|}
\hline \multirow{3}{*}{ Compounds ${ }^{a}$} & & \multicolumn{2}{|c|}{$12 \sim 33$} & \multirow{2}{*}{\multicolumn{2}{|c|}{$\mathrm{IC}_{50} \mu \mathrm{M} \pm \mathrm{SEM}$}} \\
\hline & \multirow{2}{*}{ m } & $\mathrm{n}$ & $\mathbf{R}$ & & \\
\hline & & & & $\mathrm{AChE}^{\mathrm{b}} \pm \mathrm{SEM}$ & $\mathrm{BuChE}^{\mathrm{c}} \pm \mathrm{SEM}$ \\
\hline 12 & 0 & 1 & $-N^{\prime}$ & d & $91.68 \pm 15.68$ \\
\hline 13 & 0 & 1 & $-N$ & d & $71.68 \pm 3.01$ \\
\hline 14 & 0 & 1 & $-N$ & d & $1.44 \pm 0.23$ \\
\hline 15 & 0 & 2 & $-N$ & $1.18 \pm 0.29$ & $0.74 \pm 0.09$ \\
\hline 16 & 0 & 2 & -1 & $0.88 \pm 0.17$ & $2.83 \pm 0.50$ \\
\hline 17 & 0 & 2 & -1 & $0.71 \pm 0.06$ & $2.39 \pm 0.75$ \\
\hline 18 & 0 & 2 & -1 & $0.33 \pm 0.06$ & $1.16 \pm 0.09$ \\
\hline 19 & 0 & 2 & -1 & $0.096 \pm 0.006$ & $1.25 \pm 0.15$ \\
\hline 20 & 0 & 2 & $-N$ & $0.97 \pm 0.08$ & $0.72 \pm 0.10$ \\
\hline 21 & 0 & 2 & & $1.43 \pm 0.33$ & $1.94 \pm 0.07$ \\
\hline 22 & 0 & 2 & $-\mathrm{N}$ & $1.61 \pm 0.38$ & $13.77 \pm 0.81$ \\
\hline 23 & 0 & 3 & $-N$ & $1.32 \pm 0.43$ & $5.9 \pm 0.40$ \\
\hline 24 & 0 & 4 & $-N$ & $0.82 \pm 0.16$ & $2.65 \pm 0.11$ \\
\hline 25 & 2 & 1 & $-N^{\prime}$ & $62.27 \pm 14.46$ & $9.11 \pm 2.55$ \\
\hline 26 & 2 & 1 & $-N$ & $74.54 \pm 8.50$ & $20.72 \pm 5.44$ \\
\hline 27 & 2 & 1 & $-\mathrm{N}$ & $74.13 \pm 11.53$ & $66.51 \pm 17.04$ \\
\hline 28 & 2 & 1 & $-N$ & d & $6.30 \pm 1.60$ \\
\hline 29 & 2 & 2 & -1 & $2.62 \pm 0.63$ & $1.21 \pm 0.22$ \\
\hline 30 & 2 & 2 & $-N$ & $0.50 \pm 0.06$ & $3.14 \pm 0.24$ \\
\hline 31 & 2 & 2 & $-N$ & $3.53 \pm 0.79$ & $6.38 \pm 1.26$ \\
\hline 32 & 2 & 3 & $-N$ & $3.67 \pm 0.92$ & $19.18 \pm 4.78$ \\
\hline 33 & 2 & 4 & $-N$ & $0.19 \pm 0.08$ & $1.64 \pm 0.27$ \\
\hline 34 & 0 & 2 & $-N$ & $31.74 \pm 10.24$ & $13.78 \pm 1.59$ \\
\hline 35 & 0 & 2 & $-N$ & d & d \\
\hline 36 & 2 & 2 & $-N$ & $74.93 \pm 35.56$ & $25.94 \pm 6.81$ \\
\hline Donepezil & & & & 0.044 & \\
\hline ISO-OMPA & & & & & 0.72 \\
\hline
\end{tabular}

SEM: Standard error of the mean. a The structure of compounds 12-36 shown in Scheme $1 .{ }^{b} 50 \%$ inhibitory concentration (means \pm SEM of three experiments using AChE from rat brain). ${ }^{c} 50 \%$ inhibitory concentration (means \pm SEM of three experiments using BuChE from human serum). ${ }^{\mathrm{d}}$ Concentrations of the compounds up to $100 \mu \mathrm{M} / \mathrm{L}$ were inactive. 


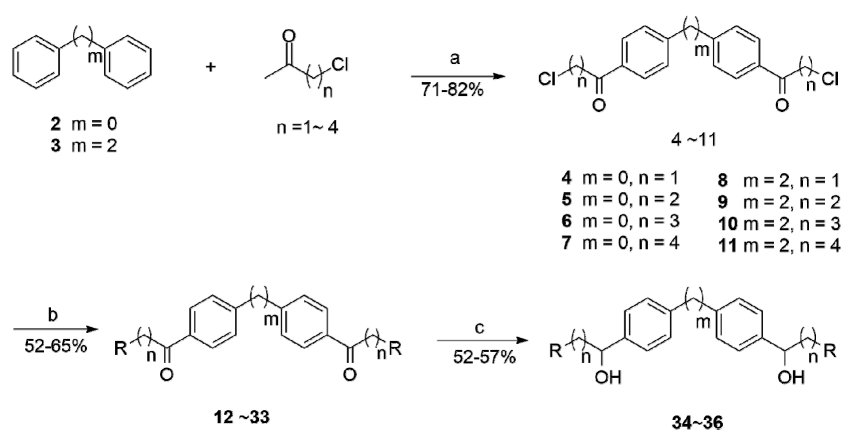

Scheme 1. Synthetic route for target compounds 12-36. Reagents, conditions, and yields: (a) $\mathrm{Cl}\left(\mathrm{CH}_{2}\right)_{\mathrm{n}} \mathrm{COCl}, \mathrm{CS}_{2} ; 40-50{ }^{\circ} \mathrm{C}, 4-8 \mathrm{~h} ; 71 \%-82 \%$; (b) secondary amines or heterocyclicamines, $\mathrm{CH}_{3} \mathrm{CN}, \mathrm{K}_{2} \mathrm{CO}_{3}, \mathrm{KI} ; 50{ }^{\circ} \mathrm{C}, 20 \mathrm{~min} ; 52 \%-65 \%$; (c) $\mathrm{NaBH}_{4}, \mathrm{THF} ; 50{ }^{\circ} \mathrm{C}, 6 \mathrm{~h} ; 52 \%-57 \%$.

\subsection{Biological Evaluation and SAR Analysis}

The abilities of the target compounds (12-36) to inhibit AChE (from rat brain) and BuChE (from human serum) activities were evaluated in vitro using the spectrophotometric method of Ellman [16]. Donepezil and tetraisopropyl-pyrophosph-oramide (iso-OMPA) were used as reference compounds, and each test was conducted in triplicate. The $\mathrm{IC}_{50}$ values are summarized in Table 1 . We found that most of the compounds showed good inhibition against both AChE and BuChE activities, with $\mathrm{IC}_{50}$ values ranging from micromolar to sub-micromolar levels. Among these compounds (12-36), compound 19 showed the strongest inhibition against $\mathrm{AChE}$, with an $\mathrm{IC}_{50}$ value of $0.096 \mu \mathrm{M}$, approximately half the potency of donepezil $\left(\mathrm{IC}_{50}=0.044 \mu \mathrm{M}\right)$. Compound 19 also exhibited potent inhibition of $\mathrm{BuChE}\left(\mathrm{IC}_{50}=1.25 \mu \mathrm{M}\right)$ which was higher than donepezil. These results indicated that compound 19 was a potent dual inhibitor against $\mathrm{AChE}$ and BuChE. We also found that compound 15 exhibited the strongest inhibition against $\mathrm{BuChE}$, with $\mathrm{IC}_{50}$ values of $0.74 \mu \mathrm{M}$, which was as potent as the reference compound iso-OPMA $\left(\mathrm{IC}_{50}=0.72 \mu \mathrm{M}\right)$; additionally, compound 15 was a potent $\mathrm{AChE}$ inhibitor $\left(\mathrm{IC}_{50}=1.18 \mu \mathrm{M}\right)$.

For all compounds, the length of linker between biphenylcarbonyl or bibenzylcarbonyl and the tertiary amine moiety played a significant role in their inhibitory activities against both AChE and $\mathrm{BuChE}$. The results indicated that a linker length of one carbon atom was not preferred. Compounds with such linker length (12-14, 25-28) showed no activity, except for compounds $\mathbf{1 4}, \mathbf{2 5}$, and $\mathbf{2 8}$. These three compounds displayed low potency for BuChE, with $\mathrm{IC}_{50}$ values of $1.44 \mu \mathrm{M}, 9.11 \mu \mathrm{M}$, and $6.30 \mu \mathrm{M}$, respectively. For the biphenyl derivatives, the biological assay showed that their $\mathrm{AChE}$ and $\mathrm{BuChE}$ inhibitory activities decreased as the length of linker increased from one carbon atom to three or four carbon atoms, although compound $\mathbf{2 4}$, containing a four-carbon linker, was only slightly less potent $\left(\mathrm{IC}_{50}=0.82 \mu \mathrm{M}\right.$ for AChE) than compound 19, with a two-carbon linker ( $\mathrm{IC}_{50}=0.096 \mu \mathrm{M}$ for AChE). These results suggested that a linker length of two carbon atoms was optimal for improving AChE inhibitory activities in compounds with a biphenyl moiety.

By contrast, the linker length for the bibenzyl derivatives made little difference with respect to their ability to inhibit $\mathrm{AChE}$ activity. For example, bibenzyl derivative $33\left(\mathrm{IC}_{50}=0.19 \mu \mathrm{M}\right.$ for AChE), with a four-carbon linker, displayed similar inhibitory activity to that for compound 19 (IC I $_{50}=0.096 \mu \mathrm{M}$ for $\mathrm{AChE})$, having a two-carbon linker. Furthermore, replacing the dimethylamine moiety in compound 1 with a heterocycloamine, such as piperidine, pyrrolidine, or azetidine, enhanced the inhibition, especially for compound 19, which was eight-fold more potent than the lead compound (1) for inhibiting AChE, while its ability to inhibit BuChE activity was only mildly decreased. Compared with biphenyl derivatives, the corresponding bibenzyl derivatives generally displayed less potent inhibition against both $\mathrm{AChE}$ and $\mathrm{BuChE}$. Finally, it appeared that the carbonyl groups in the molecules were indispensable. Reducing compounds 19, 20, and 30 to the corresponding alcohols (34-36) caused them all to lose their ability to inhibit both AChE and BuChE. 


\subsection{Molecular Docking}

To further investigate 19, the most potent compound against $\mathrm{AChE}$ and BuChE, a molecular docking study was performed, and the different types of interactions between 19 and AChE and BuChE are shown in Figures 2 and 3. As shown in Figure 2, compound 19 was located on the long tunnel of the active site interacting with some residues. To be more specific, the aromatic structure of compound 19 adopted an appropriate orientation for its binding to the phenyl ring of TYR334 and PHE330 via $\pi-\pi$ stacking interaction. In addition to the hydrophobic interaction, we can also see two key amino residues (PHE288 and ARG289) forming hydrogen bonds with the carbonyl group. All of these results indicated that compound 19 could bind to AChE.

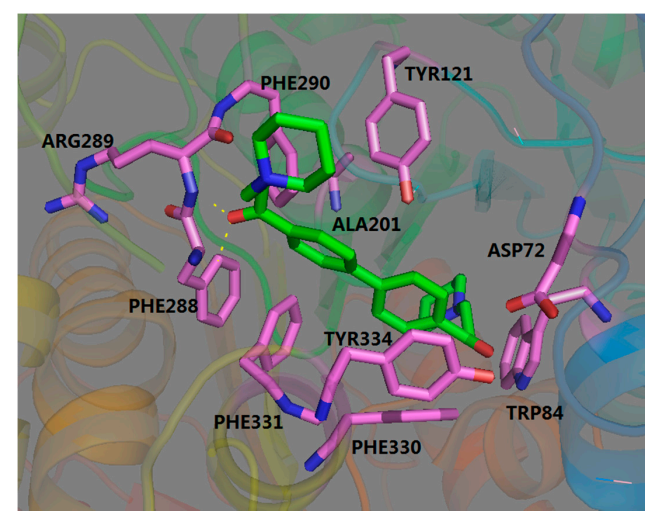

Figure 2. Three-dimensional docking model of compound 19 with AChE.

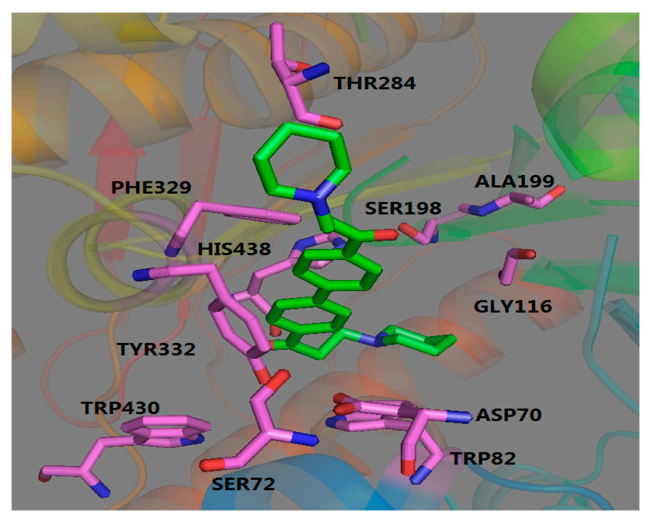

Figure 3. Three-dimensional docking model of compound 19 with BuChE.

Compare to $\mathrm{AChE}, \mathrm{BuChE}$ displayed a strong hydrophobic interaction with compound 19. As shown in Figure 3, the symmetric piperidine ring could insert into the hydrophobic cavities as well. One interaction consisted of HIS438 and TRP82, and the other was formed by THR284, PHE329. Furthermore, the aromatic structure of compound 19 adopted an appropriate orientation for its binding to the phenyl ring of TYR332 via a $\pi-\pi$ stacking interaction as well. These results illustrated that compound 19 also can bind to BuChE.

\section{Materials and Methods}

\subsection{Chemistry}

Melting points were measured on a YRT-3 apparatus (Tianjin precision apparatus factory, Tian Jin, China), uncorrected. ${ }^{1} \mathrm{H}-\mathrm{NMR}$ spectra were recorded on a Varain Mercury-300 \& 400 instrument (Varian, Salt Lake City, UT, USA) using tetramethylsilane as an internal standard, DMSO- $d_{6}$ and $\mathrm{CDCl}_{3}$ 
as solvents, chemical shifts $(\delta)$ in ppm, coupling constants $(J)$ in Hz. High-resolution mass spectra were determined with Thermo Scientific Exactive Plus mass spectrometry (Thermo Scientific, Waltham, MA, USA) with the ESI method. Column chromatography was carried out using 200-300 mesh silica gel (Qingdao Marine Chemical Inc., Qingdao, China,). All chemicals and solvents were analytical reagent grade and were used without further purification. All tested compounds were purified until the purity $\geq 99 \%$ detected by HPLC (Shimadzu Co., Kyoto, Japan) under a wavelength of 254 nm, NMR.

\subsubsection{General Method for Synthesis of Friedel-Crafts Acylation Intermediates (4-11)}

A mixture of commercial available biphenyl (2) or bibenzyl (3) $(1.0 \mathrm{mmol})$ and $\mathrm{AlCl}_{3}(3.0 \mathrm{mmol})$ was stirred in dry $\mathrm{CS}_{2}(60 \mathrm{~mL})$, and followed by $\mathrm{Cl}\left(\mathrm{CH}_{2}\right)_{\mathrm{n}} \mathrm{COCl}(n=1-4)(3.0 \mathrm{mmol})$ was added drop-wise. The reaction mixture was stirred at $50-80{ }^{\circ} \mathrm{C}$ for $4-8 \mathrm{~h}$. Then the reaction mixture was poured into $200 \mathrm{~mL}$ ice water, and the solid compound was precipitated. After washing with cold ethanol, the solid was dried to afford compounds (4-11) with the yield of $71 \%-82 \%$.

\subsubsection{General Method for Synthesis of the Target Compounds (12-33)}

A mixture of secondary alkyl amines or heterocyclic amines $(3.0 \mathrm{mmol})$, anhydrous $\mathrm{K}_{2} \mathrm{CO}_{3}$ $(4.0 \mathrm{mmol})$ and $\mathrm{KI}(1.0 \mathrm{mmol})$ was stirred in acetonitrile $(40 \mathrm{~mL})$ at $50{ }^{\circ} \mathrm{C}$ for $30 \mathrm{~min}$. Then the Friedel-Crafts acylation intermediates (4-11) was added and stirred for $20 \mathrm{~min}$. The reaction mixture was filtered and concentrated. After washing with water, the mixture was filtered and subjected to silica gel column chromatography with $\mathrm{CH}_{2} \mathrm{Cl}_{2}: \mathrm{MeOH}=100: 1$ as an eluent to afford the target compounds (12-33) with the yield of $52 \%-65 \%$.

1,1'-([1,1'-Biphenyl]-4,4'-diyl)bis(2-(dimethylamino)ethanone) (12). Yield, 52\%; m.p. 113.7-114.6 ${ }^{\circ} \mathrm{C}$; ${ }^{1} \mathrm{H}-\mathrm{NMR}\left(300 \mathrm{MHz}, \mathrm{CDCl}_{3}\right) \delta 8.10(\mathrm{~d}, J=8.1,4 \mathrm{H}), 7.70(\mathrm{~d}, J=8.1,4 \mathrm{H}), 3.79(\mathrm{~s}, 4 \mathrm{H}), 2.41(\mathrm{~s}, 12 \mathrm{H})$; ${ }^{13} \mathrm{C}-\mathrm{NMR}\left(75 \mathrm{MHz}, \mathrm{CDCl}_{3}\right): \delta 199.0,144.4,136.8,129.0,127.6,49.5,36.9 ; \mathrm{HRMS}_{(\mathrm{ESI}}{ }^{+}$) calculated for $\mathrm{C}_{20} \mathrm{H}_{25} \mathrm{~N}_{2} \mathrm{O}_{2}: 325.1916[\mathrm{M}+\mathrm{H}]^{+}$, found: 325.1903 .

1,1'-([1,1'-Biphenyl]-4,4'-diyl)bis(2-(piperidin-1-yl)ethanone) (13). Yield, 55\%; m.p. $114.3-115.4{ }^{\circ} \mathrm{C}$; ${ }^{1} \mathrm{H}-\mathrm{NMR}\left(400 \mathrm{MHz}, \mathrm{CDCl}_{3}\right) \delta 8.07(\mathrm{~d}, J=7.8,4 \mathrm{H}), 7.64(\mathrm{~d}, J=7.8,4 \mathrm{H}), 3.65(\mathrm{~s}, 4 \mathrm{H}), 3.42(\mathrm{~m}, 8 \mathrm{H}), 1.63$ $(\mathrm{m}, 12 \mathrm{H}) ;{ }^{13} \mathrm{C}-\mathrm{NMR}\left(75 \mathrm{MHz}, \mathrm{CDCl}_{3}\right): \delta 196.3,143.4,136.5,129.8,127.1,65.2,54.7,33.0,22.5$; HRMS (ESI+) calculated for $\mathrm{C}_{26} \mathrm{H}_{33} \mathrm{~N}_{2} \mathrm{O}_{2}: 405.2542[\mathrm{M}+\mathrm{H}]^{+}$, found: 405.2544 .

1,1'-([1,1'-Biphenyl]-4,4'-diyl)bis(2-morpholinoethanone) (14). Yield, 65\%; m.p. 112.2-113.5 ${ }^{\circ} \mathrm{C} ;{ }^{1} \mathrm{H}-\mathrm{NMR}$ $\left(300 \mathrm{MHz}, \mathrm{CDCl}_{3}\right) \delta 8.04(\mathrm{~d}, J=8.4,4 \mathrm{H}), 7.74(\mathrm{~d}, J=8.4,4 \mathrm{H}), 3.78(\mathrm{~s}, 4 \mathrm{H}), 3.72(\mathrm{t}, J=4.5,8 \mathrm{H}), 2.57(\mathrm{t}$, $J=4.5,8 \mathrm{H}) ;{ }^{13} \mathrm{C}-\mathrm{NMR}\left(125 \mathrm{MHz} \mathrm{CDCl}_{3}\right): \delta 195.8,144.7,135.5,129.1,127.7,67.0,65.0,54.1$; HRMS (ESI+) calculated for $\mathrm{C}_{24} \mathrm{H}_{29} \mathrm{~N}_{2} \mathrm{O}_{4}: 409.2127[\mathrm{M}+\mathrm{H}]^{+}$, found: 409.2123 .

4,4'-Bis( $\beta$-diethylamino-propinoyl)-biphenyl (15). Yield, 57\%; m.p. $129.2-130.6{ }^{\circ} \mathrm{C} ;{ }^{1} \mathrm{H}-\mathrm{NMR}(400 \mathrm{MHz}$, DMSO-d $\left.d_{6}\right) \delta 8.15(\mathrm{~d}, 4 \mathrm{H}, J=8.4), 7.99(\mathrm{~d}, 4 \mathrm{H}, J=8.4), 3.71(\mathrm{t}, 4 \mathrm{H}, J=6.9), 3.43(\mathrm{t}, 4 \mathrm{H}, J=6.9), 3.22(\mathrm{q}, 8 \mathrm{H}$, $J=7.2), 1.270(\mathrm{t}, 12 \mathrm{H}, J=7.2) ;{ }^{13} \mathrm{C}-\mathrm{NMR}\left(75 \mathrm{MHz}, \mathrm{DMSO}-d_{6}\right): \delta 197.2,144.2,132.77,129.0,127.6,47.6$, 42.0, 33.9, 11.4; HRMS (ESI+) calculated for $\mathrm{C}_{26} \mathrm{H}_{27} \mathrm{~N}_{2} \mathrm{O}_{2}: 409.2855[\mathrm{M}+\mathrm{H}]^{+}$, found: 409.2845 .

4,4'-Bis( $\beta$-dipropylamino-propinoyl)-biphenyl (16). Yield, 52\%; m.p. 131.0-132.6 ${ }^{\circ} \mathrm{C} ;{ }^{1} \mathrm{H}-\mathrm{NMR}(400 \mathrm{MHz}$, $\left.\mathrm{CDCl}_{3}\right) \delta 8.06(\mathrm{~d}, 4 \mathrm{H}, J=8.4), 7.71(\mathrm{~d}, 4 \mathrm{H}, J=8.4), 3.14(\mathrm{t}, 4 \mathrm{H}, J=6.8), 2.94(\mathrm{t}, 4 \mathrm{H}, J=6.8), 2.42(\mathrm{t}$, $8 \mathrm{H}, J=7.6), 1.47(\mathrm{~m}, 8 \mathrm{H}), 0.871(\mathrm{t}, 12 \mathrm{H}, J=7.6) ;{ }^{13} \mathrm{C}-\mathrm{NMR}\left(75 \mathrm{MHz}, \mathrm{CDCl}_{3}\right): \delta 199.7,144.4,136.8$, 129.0, 127.6, 56.4, 49.5, 36.9, 20.6, 12.1; HRMS (ESI+) calculated for $\mathrm{C}_{30} \mathrm{H}_{45} \mathrm{~N}_{2} \mathrm{O}_{2}: 465.3481[\mathrm{M}+\mathrm{H}]^{+}$, found: 465.3479 .

4,4'-Bis( $\beta$-azetidin-propinoyl)-biphenyl (17). Yield, 60\%; m.p. 150.8-151.1 ${ }^{\circ} \mathrm{C} ;{ }^{1} \mathrm{H}-\mathrm{NMR}\left(400 \mathrm{MHz}, \mathrm{CDCl}_{3}\right)$ $\delta 8.05(\mathrm{~d}, 4 \mathrm{H}, J=8.0), 7.71(\mathrm{~d}, 4 \mathrm{H}, J=8.0), 3.26(\mathrm{t}, 8 \mathrm{H}, J=6.8), 3.05(\mathrm{t}, 4 \mathrm{H}, J=7.2), 2.86(\mathrm{t}, 4 \mathrm{H}, J=7.2)$, $2.09(\mathrm{t}, 4 \mathrm{H}, \mathrm{J}=6.8) ;{ }^{13} \mathrm{C}-\mathrm{NMR}\left(75 \mathrm{MHz} \mathrm{CDCl}_{3}\right): \delta 198.4,144.3,136.3,128.8,127.5,55.3,54.5,37.0,17.6$; HRMS (ESI+) calculated for $\mathrm{C}_{24} \mathrm{H}_{29} \mathrm{~N}_{2} \mathrm{O}_{2}$ : $377.2229[\mathrm{M}+\mathrm{H}]^{+}$, found: 377.2218. 
4,4'-Bis( $\beta$-pyrrolidin-propinoyl)-biphenyl (18). Yield, 62\%; m.p. 151.2-152.1 ${ }^{\circ} \mathrm{C} ;{ }^{1} \mathrm{H}-\mathrm{NMR}(300 \mathrm{MHz}$, $\left.\mathrm{CDCl}_{3},\right) \delta 8.06(\mathrm{~d}, 4 \mathrm{H}, J=8.1), 7.72(\mathrm{~d}, 4 \mathrm{H}, J=8.1), 3.28(\mathrm{t}, 4 \mathrm{H}, J=7.2), 2.97(\mathrm{t}, 4 \mathrm{H}, J=7.2), 2.62(\mathrm{~m}$, 8H), $1.824(\mathrm{~m}, 8 \mathrm{H}) ;{ }^{13} \mathrm{C}-\mathrm{NMR}\left(75 \mathrm{MHz}, \mathrm{CDCl}_{3}\right): \delta 198.4,144.2,136.3,128.8,127.5,54.3,51.0,38.2,23.5$; HRMS (ESI+) calculated for $\mathrm{C}_{26} \mathrm{H}_{33} \mathrm{~N}_{2} \mathrm{O}_{2}: 405.2542[\mathrm{M}+\mathrm{H}]^{+}$, found: 405.2535 .

4,4'-Bis( $\beta$-(piperidin-propinoyl)-biphenyl (19). Yield, 59\%; m.p. 138.5-139.6 ${ }^{\circ} \mathrm{C} ;{ }^{1} \mathrm{H}-\mathrm{NMR}(400 \mathrm{MHz}$, DMSO- $\left.d_{6}\right) \delta 8.15(\mathrm{~d}, 4 \mathrm{H}, J=8.0), 7.99(\mathrm{~d}, 4 \mathrm{H}, J=8.0), 3.66(\mathrm{t}, 4 \mathrm{H}, J=7.2), 3.55(\mathrm{t}, 4 \mathrm{H}, J=8.8), 3.44(\mathrm{t}$, $4 \mathrm{H}, J=7.2), 3.01(\mathrm{t}, 4 \mathrm{H}, J=8.8), 1.87(\mathrm{~m}, 4 \mathrm{H}), 1.71(\mathrm{~m}, 4 \mathrm{H}), 1.41(\mathrm{~m}, 4 \mathrm{H}) ;{ }^{13} \mathrm{C}-\mathrm{NMR}\left(125 \mathrm{MHz}, \mathrm{CDCl}_{3}\right)$ : $\delta$ 196.3, 143.4, 135.5, 128.8, 127.4, 52.2, 51.1, 33.0, 22.5, 21.3; HRMS (ESI+) calculated for $\mathrm{C}_{28} \mathrm{H}_{37} \mathrm{~N}_{2} \mathrm{O}_{2}$ $[\mathrm{M}+\mathrm{H}]^{+}:$433.2855, found: 433.2844 .

4,4'-Bis( $\beta$-(morpholino-propinoyl)-biphenyl (20). Yield, 58\%; m.p. 140.1-141.0 ${ }^{\circ} \mathrm{C} ;{ }^{1} \mathrm{H}-\mathrm{NMR}(400 \mathrm{MHz}$, $\left.\mathrm{CDCl}_{3}\right) \delta 8.06(\mathrm{~d}, J=8.0,4 \mathrm{H}), 7.72(\mathrm{~d}, J=8.0,4 \mathrm{H}), 3.76(\mathrm{~m}, 8 \mathrm{H}), 3.22(\mathrm{t}, J=7.2,4 \mathrm{H}), 2.86(\mathrm{t}, J=7.2$, $4 \mathrm{H}), 2.53(\mathrm{~m}, 8 \mathrm{H}) ;{ }^{13} \mathrm{C}-\mathrm{NMR}\left(125 \mathrm{MHz}, \mathrm{CDCl}_{3}\right): \delta 198.4,144.6,136.4,129.0,127.7,66.9,53.9,53.7,36.1$; HRMS (ESI+) calculated for $\mathrm{C}_{26} \mathrm{H}_{33} \mathrm{~N}_{2} \mathrm{O}_{4}: 437.2440[\mathrm{M}+\mathrm{H}]^{+}$, found: 437.2431.

4,4'-Bis( $\beta$-azepan-propinoyl)-biphenyl (21). Yield, 52\%; m.p. 109.2-111.5 ${ }^{\circ} \mathrm{C} ;{ }^{1} \mathrm{H}-\mathrm{NMR}\left(400 \mathrm{MHz}, \mathrm{CDCl}_{3}\right)$ $\delta 8.05(\mathrm{~d}, 4 \mathrm{H}, J=8.4), 7.70(\mathrm{~d}, 4 \mathrm{H}, J=8.4), 3.23(\mathrm{t}, 4 \mathrm{H}, J=7.6), 3.03(\mathrm{t}, 4 \mathrm{H}, J=7.6), 2.74(\mathrm{t}, 8 \mathrm{H}, J=5.6)$, $1.68(\mathrm{~m}, 8 \mathrm{H}), 1.59(\mathrm{~m}, 8 \mathrm{H}) ;{ }^{13} \mathrm{C}-\mathrm{NMR}\left(75 \mathrm{MHz} \mathrm{CDCl}_{3}\right): \delta 199.1,144.4,136.5,128.9,127.6,55.6,53.3,37.0$, 27.7, 27.1; HRMS (ESI+) calculated for $\mathrm{C}_{30} \mathrm{H}_{41} \mathrm{~N}_{2} \mathrm{O}_{2}: 461.3168[\mathrm{M}+\mathrm{H}]^{+}$, found: 461.3158.

4,4'-Bis ( $\beta$-4-methylpiperazin-propinoyl)-biphenyl (22). Yield, 50\%; m.p. $146.1-148.7^{\circ} \mathrm{C} ;{ }^{1} \mathrm{H}-\mathrm{NMR}(400$ $\left.\mathrm{MHz}, \mathrm{CDCl}_{3}\right) \delta 8.06(\mathrm{~d}, 4 \mathrm{H}, J=8.4), 7.73(\mathrm{~d}, 4 \mathrm{H}, J=8.4), 3.23(\mathrm{t}, 4 \mathrm{H}, J=7.6), 2.89(\mathrm{t}, 4 \mathrm{H}, J=7.6), 2.59(\mathrm{~m}$, 8H), $2.48(\mathrm{~m}, 8 \mathrm{H}), 2.31(\mathrm{~s}, 6 \mathrm{H}) ;{ }^{13} \mathrm{C}-\mathrm{NMR}\left(75 \mathrm{MHz}, \mathrm{CDCl}_{3}\right): \delta$ 198.6, 144.3, 136.4, 128.7, 127.5, 55.1, 53.2, 53.1, 46.0, 36.4; HRMS (ESI+) calculated for $\mathrm{C}_{28} \mathrm{H}_{39} \mathrm{~N}_{4} \mathrm{O}_{2}[\mathrm{M}+\mathrm{H}]^{+}: 463.3073$, found: 463.3067.

1,1'-([1,1'-Biphenyl]-4,4'-diyl)bis(4-(piperidin-1-yl)butan-1-one) (23). Yield, 53\%; m.p. 146.4-147.8 ${ }^{\circ} \mathrm{C}$; ${ }^{1} \mathrm{H}-\mathrm{NMR}\left(400 \mathrm{MHz}, \mathrm{CDCl}_{3}\right) \delta 8.12(\mathrm{~d}, 4 \mathrm{H}, J=8.0), 7.78(\mathrm{~d}, 4 \mathrm{H}, J=8.0), 3.09(\mathrm{t}, 4 \mathrm{H}, J=7.2), 2.44(\mathrm{~m}$, 12H), $2.07(\mathrm{~m}, 4 \mathrm{H}), 1.59(\mathrm{~m}, 8 \mathrm{H}), 1.44(\mathrm{~m}, 4 \mathrm{H}) ;{ }^{13} \mathrm{C}-\mathrm{NMR}\left(125 \mathrm{MHz}, \mathrm{CDCl}_{3}\right): \delta$ 199.7, 144.4, 136.6, 129.0, 127.6, 58.5, 54.5, 36.7, 25.7, 24.4, 21.5. HRMS (ESI+) calculated for $\mathrm{C}_{30} \mathrm{H}_{41} \mathrm{~N}_{2} \mathrm{O}_{2}[\mathrm{M}+\mathrm{H}]^{+}: 461.3168$, found: 461.3162 .

1,1'-([1,1'-Biphenyl]-4,4'-diyl)bis(5-(piperidin-1-yl)pentan-1-one) (24). Yield, 48\%; m.p. 115.7-117.8 ${ }^{\circ} \mathrm{C}$; ${ }^{1} \mathrm{H}-\mathrm{NMR}\left(400 \mathrm{MHz}, \mathrm{CDCl}_{3}\right) \delta 8.05(\mathrm{~d}, 4 \mathrm{H}, J=8.4), 7.70(\mathrm{~d}, 4 \mathrm{H}, J=8.4), 3.03(\mathrm{t}, 4 \mathrm{H}, J=7.2), 2.45(\mathrm{~m}$, 12H), $1.80(\mathrm{~m}, 4 \mathrm{H}), 1.59(\mathrm{~m}, 12 \mathrm{H}), 1.44(\mathrm{~m}, 4 \mathrm{H}) ;{ }^{13} \mathrm{C}-\mathrm{NMR}\left(125 \mathrm{MHz}, \mathrm{CDCl}_{3}\right): \delta$ 199.7, 144.4, 136.6, 129.0, 127.6, 58.5, 54.5, 36.7, 25.7, 24.4, 21.5; HRMS (ESI+) calculated for $\mathrm{C}_{32} \mathrm{H}_{45} \mathrm{~N}_{2} \mathrm{O}_{2}[\mathrm{M}+\mathrm{H}]^{+}: 489.3481$; found, 489.3473 .

1,1'-(Ethane-1,2-diylbis(4,1-phenylene))bis(2-(dimethylamino)ethanone) (25). Yield, 58\%; m.p. 104.2-106.9 ${ }^{\circ} \mathrm{C} ;{ }^{1} \mathrm{H}-\mathrm{NMR}\left(300 \mathrm{MH}_{\mathrm{Z}}, \mathrm{CDCl}_{3}\right) \delta 7.91(\mathrm{~d}, 4 \mathrm{H}, J=8.1), 7.22(\mathrm{~d}, 4 \mathrm{H}, J=8.1), 3.75(\mathrm{~s}, 4 \mathrm{H}), 2.99(\mathrm{~s}, 4 \mathrm{H})$, 2.39 (s, 12H). ${ }^{13} \mathrm{C}-\mathrm{NMR}\left(125 \mathrm{MHz}, \mathrm{CDCl}_{3}\right): \delta 196.2,147.2,134.2,130.0,128.9,65.3,45.9,37.5$; HRMS (ESI+): calculated for $\mathrm{C}_{22} \mathrm{H}_{29} \mathrm{~N}_{2} \mathrm{O}_{2}[\mathrm{M}+\mathrm{H}]^{+}: 353.2229$, found: 353.2217 .

1,1'-(Ethane-1,2-diylbis(4,1-phenylene))bis(2-(piperidin-1-yl)ethanone) (26). Yield, 52\%; m.p. 96.7-98.6 ${ }^{\circ} \mathrm{C}$; ${ }^{1} \mathrm{H}-\mathrm{NMR}\left(300 \mathrm{MHz}, \mathrm{CDCl}_{3}\right) \delta 7.92(\mathrm{~d}, 4 \mathrm{H}, J=7.8), 7.21(\mathrm{~d}, 4 \mathrm{H}, J=7.8), 3.79(\mathrm{~s}, 4 \mathrm{H}), 2.99(\mathrm{~s}, 4 \mathrm{H}), 2.58(\mathrm{~m}$, $8 \mathrm{H}), 1.67(\mathrm{~m}, 8 \mathrm{H}), 1.47(\mathrm{~m}, 4 \mathrm{H}) ;{ }^{13} \mathrm{C}-\mathrm{NMR}\left(125 \mathrm{MHz}, \mathrm{CDCl}_{3}\right): \delta 196.7,147.0,134.6,128.8,128.6,65.5$, 55.1, 37.6, 26.0, 24.2; HRMS (ESI+) calculated for $\mathrm{C}_{28} \mathrm{H}_{37} \mathrm{~N}_{2} \mathrm{O}_{2}[\mathrm{M}+\mathrm{H}]^{+}, 433.2855$, found: 433.2850 .

1,1'-(Ethane-1,2-diylbis(4,1-phenylene))bis(2-(4-methylpiperazin-1-yl)ethanone) (27). Yield, 54\%; m.p. 150.9-152.2 ${ }^{\circ} \mathrm{C} ;{ }^{1} \mathrm{H}-\mathrm{NMR}\left(300 \mathrm{MHz}, \mathrm{CDCl}_{3}\right) \delta 7.91(\mathrm{~d}, 4 \mathrm{H}, J=8.1), 7.223(\mathrm{~d}, 4 \mathrm{H}, J=8.1), 3.79(\mathrm{~s}$, $4 \mathrm{H}), 2.99(\mathrm{~s}, 4 \mathrm{H}), 2.64(\mathrm{~m}, 8 \mathrm{H}), 2.530(\mathrm{~m}, 8 \mathrm{H}) 2.31(\mathrm{~s}, 6 \mathrm{H}) ;{ }^{13} \mathrm{C}-\mathrm{NMR}\left(125 \mathrm{MHz}, \mathrm{CDCl}_{3}\right): \delta$ 195.9, 147.1, 134.3, 128.9, 128.5, 64.4, 55.0, 53.4, 46.0, 37.5; HRMS (ESI+) calculated for $\mathrm{C}_{28} \mathrm{H}_{39} \mathrm{~N}_{4} \mathrm{O}_{2}[\mathrm{M}+\mathrm{H}]^{+}$: 463.3073, found: 463.3066 .

1,1'-(Ethane-1,2-diylbis(4,1-phenylene))bis(5-morpholinopentan-1-one) (28). Yield, 52\%; m.p. 160.1-162.6 ${ }^{\circ} \mathrm{C}$; ${ }^{1} \mathrm{H}-\mathrm{NMR}\left(300 \mathrm{MHz}, \mathrm{CDCl}_{3}\right) \delta 7.91(\mathrm{~d}, 4 \mathrm{H}, J=8.4), 7.23(\mathrm{~d}, 4 \mathrm{H}, J=8.4), 3.79(\mathrm{~m}, 12 \mathrm{H}), 3.01(\mathrm{~s}, 4 \mathrm{H}), 2.63$ 
(m, 8H); ${ }^{13} \mathrm{C}-\mathrm{NMR}\left(125 \mathrm{MHz}, \mathrm{CDCl}_{3}\right): \delta$ 195.8, 147.2, 134.3, 128.9, 128.5, 67.0, 64.8, 54.1, 37.5; HRMS (ESI+) calculated for $\mathrm{C}_{28} \mathrm{H}_{33} \mathrm{~N}_{2} \mathrm{O}_{4}[\mathrm{M}+\mathrm{H}]^{+}:$437.2440, found: 437.2434.

1,1'-(Ethane-1,2-diylbis(4,1-phenylene))bis(3-(dimethylamino)propan-1-one) (29). Yield, 56\%; m.p. 94.5-95.6 ${ }^{\circ} \mathrm{C} ;{ }^{1} \mathrm{H}-\mathrm{NMR}\left(400 \mathrm{MHz}, \mathrm{CDCl}_{3}\right) \delta 7.91(\mathrm{~d}, 4 \mathrm{H}, J=8.0 \mathrm{~Hz}), 7.25(\mathrm{~d}, 4 \mathrm{H}, J=8.0), 3.14(\mathrm{t}, 4 \mathrm{H}, J=7.2), 2.99$ (s, 4H), $2.77(\mathrm{t}, 4 \mathrm{H}, J=7.2), 2.24(\mathrm{~s}, 12 \mathrm{H}) ;{ }^{13} \mathrm{C}-\mathrm{NMR}\left(125 \mathrm{MHz}, \mathrm{CDCl}_{3}\right): \delta$ 198.6, 147.0, 135.1, 129.0, 128.5, 54.4, 45.6, 37.5, 36.8; HRMS (ESI+) calculated for $\mathrm{C}_{24} \mathrm{H}_{33} \mathrm{~N}_{2} \mathrm{O}_{2}[\mathrm{M}+\mathrm{H}]^{+}: 381.2545$, found: 381.2542.

1,1'-(Ethane-1,2-diylbis(4,1-phenylene))bis(3-(piperidin-1-yl)propan-1-one) (30). Yield, 52\%; m.p. 103.5-104.8 ${ }^{\circ} \mathrm{C} ;{ }^{1} \mathrm{H}-\mathrm{NMR}\left(400 \mathrm{MHz}, \mathrm{CDCl}_{3}\right) \delta 7.86(\mathrm{~d}, J=8.2,4 \mathrm{H}), 7.21(\mathrm{~d}, J=8.2,4 \mathrm{H}), 3.10(\mathrm{~m}, 4 \mathrm{H}), 2.99(\mathrm{~s}, 4 \mathrm{H})$, $2.79(\mathrm{t}, J=7.5,4 \mathrm{H}), 2.45(\mathrm{~s}, 8 \mathrm{H}), 1.60(\mathrm{~m}, 8 \mathrm{H}), 1.44(\mathrm{~d}, J=4.8,4 \mathrm{H}) ;{ }^{13} \mathrm{C}-\mathrm{NMR}\left(125 \mathrm{MHz}, \mathrm{CDCl}_{3}\right): \delta$ 199.0, 146.9, 135.2, 129.0, 128.5, 54.8, 54.0, 37.5, 36.3, 26.0, 24.3; HRMS (ESI+) calculated for $\mathrm{C}_{30} \mathrm{H}_{40} \mathrm{~N}_{2} \mathrm{O}_{2}$ $[\mathrm{M}+\mathrm{H}]^{+}: 461.3168$, found: 461.3162 .

1,1'-(Ethane-1,2-diylbis(4,1-phenylene))bis(3-morpholinopropan-1-one) (31). Yield, 56\%; m.p. 105.2-106.6 ${ }^{\circ} \mathrm{C}$; ${ }^{1} \mathrm{H}-\mathrm{NMR}\left(400 \mathrm{MHz}, \mathrm{CDCl}_{3}\right) \delta 7.86(\mathrm{~d}, J=8.2,4 \mathrm{H}), 7.22(\mathrm{~d}, J=8.1,4 \mathrm{H}), 3.75-3.66(\mathrm{~m}, 8 \mathrm{H}), 3.17(\mathrm{t}, J=7.3$, $4 \mathrm{H}), 3.00(\mathrm{~s}, 4 \mathrm{H}), 2.84(\mathrm{t}, J=7.3,4 \mathrm{H}), 2.53(\mathrm{~s}, 8 \mathrm{H}) ;{ }^{13} \mathrm{C}-\mathrm{NMR}\left(125 \mathrm{MHz}, \mathrm{CDCl}_{3}\right): \delta 198.7,147.0,135.2$, 129.0, 128.5, 67.1, 53.9 53.8, 37.5, 36.1; HRMS (ESI+) calculated for $\mathrm{C}_{28} \mathrm{H}_{36} \mathrm{~N}_{2} \mathrm{O}_{4}[\mathrm{M}+\mathrm{H}]^{+}: 465.2753$, found: 465.2744 .

1,1'-(Ethane-1,2-diylbis(4,1-phenylene))bis(4-(piperidin-1-yl)butan-1-one) (32). Yield, 53\%; m.p. 145.2-146.6 ${ }^{\circ} \mathrm{C} ;{ }^{1} \mathrm{H}-\mathrm{NMR}\left(400 \mathrm{MHz}, \mathrm{CDCl}_{3}\right) \delta 7.87(\mathrm{~d}, 4 \mathrm{H}, J=8.4), 7.21(\mathrm{~d}, 4 \mathrm{H}, J=8.4), 2.99(\mathrm{~s}, 4 \mathrm{H}), 2.95(\mathrm{t}, 4 \mathrm{H}$, $J=7.2), 2.32(\mathrm{~m}, 12 \mathrm{H}), 1.86(\mathrm{~m}, 4 \mathrm{H}), 1.48(\mathrm{~m}, 8 \mathrm{H}), 1.35(\mathrm{~m}, 4 \mathrm{H}) ;{ }^{13} \mathrm{C}-\mathrm{NMR}\left(125 \mathrm{MHz}, \mathrm{CDCl}_{3}\right): \delta 199.9$, 146.7, 135.5, 129.0, 128.5, 58.7, 54.6, 37.5, 36.6, 25.9, 24.5, 21.6; HRMS (ESI+) calculated for $\mathrm{C}_{32} \mathrm{H}_{45} \mathrm{~N}_{2} \mathrm{O}_{2}$ $[\mathrm{M}+\mathrm{H}]^{+}, 488.3481$, found: 488.3475 .

1,1'-(Ethane-1,2-diylbis(4,1-phenylene))bis(5-(piperidin-1-yl)pentan-1-one) (33). Yield, 68\%; m.p. 146.1-147.5 ${ }^{\circ} \mathrm{C} ;{ }^{1} \mathrm{H}-\mathrm{NMR}\left(300 \mathrm{MHz}, \mathrm{CDCl}_{3}\right) \delta 7.87(\mathrm{~d}, 4 \mathrm{H}, J=8.1 \mathrm{~Hz}), 7.21(\mathrm{~d}, 4 \mathrm{H}, J=8.1), 3.00(\mathrm{~s}, 4 \mathrm{H}), 2.97(\mathrm{t}$, $4 \mathrm{H}, J=7.2), 2.36(\mathrm{~m}, 12 \mathrm{H}), 1.74(\mathrm{~m}, 4 \mathrm{H}), 1.60(\mathrm{~m}, 12 \mathrm{H}), 1.44(\mathrm{~m}, 4 \mathrm{H}) ;{ }^{13} \mathrm{C}-\mathrm{NMR}\left(125 \mathrm{MHz}, \mathrm{CDCl}_{3}\right): \delta$ 200.1 146.7, 135.3, 128.9, 128.5, 59.2, 54.7, 38.5, 37.5, 26.6, 25.9, 24.5, 22.6; HRMS (ESI+) calculated for $\mathrm{C}_{34} \mathrm{H}_{49} \mathrm{~N}_{2} \mathrm{O}_{2}[\mathrm{M}+\mathrm{H}]^{+}$: 517.3794, found: 517.3787.

\subsubsection{General Method for Synthesis of Target Compounds (34-36)}

To a stirred solution of compounds $(\mathbf{1 9}, 20$ and 30) $(1.0 \mathrm{mmol})$ in anhydrous THF (30 mL), sodium borohydride $(3.0 \mathrm{mmol})$ was added with stirring. The reaction mixture was stirred at $50{ }^{\circ} \mathrm{C}$ for $6 \mathrm{~h}$. Then the reaction mixture was filtered and quenched by methanol. After the concentrated, the residue was subjected to silica gel column chromatography with DCM:MeOH $=100: 1$ as an eluent to afford the target alcohols (34-36) with the yield of 52\%-57\%.

1,1'-([1,1'-Biphenyl]-4,4'-diyl)bis(3-(piperidin-1-yl)propan-1-ol) (34). Yield, 52\%; m.p. 139.5-141.6 ${ }^{\circ} \mathrm{C}$; ${ }^{1} \mathrm{H}-\mathrm{NMR}\left(400 \mathrm{MHz}, \mathrm{CDCl}_{3}\right) \delta 7.57(\mathrm{~d}, 4 \mathrm{H}, J=8.4), 7.43(\mathrm{~d}, 4 \mathrm{H}, J=8.4), 4.98(\mathrm{t}, 2 \mathrm{H}, J=5.6), 2.58(\mathrm{~m}, 8 \mathrm{H})$, $2.43(\mathrm{~m}, 4 \mathrm{H}), 1.87(\mathrm{~m}, 4 \mathrm{H}), 1.64(\mathrm{~m}, 8 \mathrm{H}), 1.06(\mathrm{~m}, 4 \mathrm{H}) ;{ }^{13} \mathrm{C}-\mathrm{NMR}\left(75 \mathrm{MHz}, \mathrm{CDCl}_{3}\right): \delta$ 144.1, 139.6, 126.8, 125.9, 75.5, 57.8, 54.7, 33.6, 26.1, 24.3; HRMS (ESI+) calculated for $\mathrm{C}_{28} \mathrm{H}_{41} \mathrm{~N}_{2} \mathrm{O}_{2}[\mathrm{M}+\mathrm{H}]^{+}: 437.3168$, found: 437.3162 .

1,1'-([1,1'-Biphenyl]-4,4'-diyl)bis(3-morpholinopropan-1-ol) (35). Yield, 57\%; m.p. 131.2-133.6 ${ }^{\circ} \mathrm{C} ;{ }^{1} \mathrm{H}-\mathrm{NMR}$ $\left(400 \mathrm{MHz}, \mathrm{CDCl}_{3}\right) \delta 7.57$ (d, 4H, J = 7.6), 7.43 (d, 4H, $\left.J=7.6\right), 6.40$ (brs, 2H), 4.96 (brs, 2H), 3.75 (m, $8 \mathrm{H}), 2.65(\mathrm{~m}, 8 \mathrm{H}), 2.49(\mathrm{~m}, 4 \mathrm{H}), 1.89(\mathrm{~m}, 4 \mathrm{H}, J=4.4) ;{ }^{13} \mathrm{C}-\mathrm{NMR}\left(75 \mathrm{MHz}, \mathrm{CDCl}_{3}\right): \delta 143.6,139.5,126.7$, 125.8, 75.0, 66.8, 57.3, 53.5, 33.3; HRMS (ESI+) calculated for $\mathrm{C}_{28} \mathrm{H}_{37} \mathrm{~N}_{2} \mathrm{O}_{4}[\mathrm{M}+\mathrm{H}]^{+}: 440.2753$, found: 440.2743 .

1,1'-(Ethane-1,2-diylbis(4,1-phenylene))bis(3-(piperidin-1-yl)propan-1-ol) (36). Yield, 52\%; m.p. 141.5-142.6 ${ }^{\circ} \mathrm{C} ;{ }^{1} \mathrm{H}-\mathrm{NMR}\left(400 \mathrm{MHz}, \mathrm{CDCl}_{3}\right): \delta 7.28(\mathrm{~d}, 4 \mathrm{H}, J=8.4), 7.15(\mathrm{~d}, 4 \mathrm{H}, J=8.4), 4.90(\mathrm{t}, 2 \mathrm{H}, J=5.6) 2.89(\mathrm{~s}$, $4 \mathrm{H}), 2.57(\mathrm{~m}, 8 \mathrm{H}), 2.41(\mathrm{~m}, 4 \mathrm{H}), 1.83(\mathrm{~m}, 4 \mathrm{H}), 1.62(\mathrm{~m}, 8 \mathrm{H}), 1.46(\mathrm{~m}, 4 \mathrm{H}) ;{ }^{13} \mathrm{C}-\mathrm{NMR}\left(75 \mathrm{MHz}, \mathrm{CDCl}_{3}\right): \delta$ 
142.7, 140.3, 128.2, 125.5, 75.5, 57.7, 54.6, 37.6, 33.6, 26.0, 24.2; HRMS (ESI+) calculated for $\mathrm{C}_{30} \mathrm{H}_{45} \mathrm{~N}_{2} \mathrm{O}_{2}$ $[\mathrm{M}+\mathrm{H}]^{+}:$465.3481, found: 465.3462 .

\subsection{Biological Evaluation}

All of the assay of acetylcholinesterase (AChE) and butyrylcholinesterase (BuChE) were performed according to the method described by Ellman et al. [17] using the rat AChE purified from the rat brain and human BuChE purified by human serum. In an enzymatic reaction medium contained different concentration of tested compounds and $\mathrm{AChE}$ suspended in the phosphate buffer solution at $\mathrm{pH}$ 7.2. After $30 \mathrm{~min}$ at room temperature, acetylthiocholine iodide and 5.5'-dithiobis-2-nitrobenzoic acid (DNTB) were added and the reaction mixture incubated at $37^{\circ} \mathrm{C}$ for $60 \mathrm{~min}$. After the initiation of enzymatic reaction, absorbance of each tested compound mixture of colored end-product was measured by using the UV-VIS spectrophotometer at $412 \mathrm{~nm}$. The parallel control experiment was carried without tested compounds in the reaction mixture. For BuChE inhibitory assay, the procedure described above was followed except for the use of enzyme and substrate, instead of which butyrylcholinesterase and butyrylthiocholine chloride were used, respectively. Assays were done with a blank containing all compounds except $\mathrm{AChE}$ or $\mathrm{BuChE}$ in order to account for nonenzymatic reactions. Each inhibitory test was conducted in triplicate. Donepezil was used as a positive control of AChE and ISO-OMPA was used as a positive control of BuChE, respectively. Each concentration was assayed in triplicate and data were expressed as the mean \pm SEM.

\subsection{Molecular Docking}

To further investigate the interaction of the most active compound 19 for $\mathrm{AChE}$ and BuChE, a molecular docking study was performed by utilizing the Gold 3.0.1 software package (Cambridge Crystallographic Data Center, Cambridge, UK). The X-ray crystal structure of the AChE (PDB code: 1EVE) and BuChE (PDB code: 4BDS) were obtained from protein data bank. Discovery Studio and Pymol program were also used to prepare 2D and 3D schematic diagrams of docking model to exhibit different interaction types between 19 and $\mathrm{AChE} / \mathrm{BuChE}$.

\section{Conclusions}

In summary, a new class of diphenyl/dibenzyl derivatives (12-36) has been designed, synthesized, and determined to function as novel dual inhibitors of $\mathrm{AChE}$ and BuChE. Most of these compounds possessed moderate to high $\mathrm{AChE}$ and BuChE inhibitory activities in vitro. Compound 19 showed the most potent $\mathrm{AChE}$ inhibitory activity, in the sub-micromolar range $\left(\mathrm{IC}_{50}=0.096 \mu \mathrm{M}\right)$, and moderate BuChE inhibition, with an $\mathrm{IC}_{50}$ value of $1.25 \mu \mathrm{M}$. Compound 15 exhibited the most potent inhibition against $\mathrm{BuChE}$, with an $\mathrm{IC}_{50}$ value of $0.74 \mu \mathrm{M}$, which was as potent as the reference compound iso-OPMA. Our molecular modeling study confirmed that compound $\mathbf{1 9}$ is capable of binding to the active-site cavity of both AChE and BuchE. Among the tested compounds, compound 19 is considered to be a promising dual inhibitor against $\mathrm{AChE}$ and $\mathrm{BuChE}$ and, thus, a therapeutic candidate in $\mathrm{AD}$.

Acknowledgments: This work was supported by National Natural Science Foundation of China (for young scientists) (No. 21302228).

Author Contributions: Design, Synthesis, experimental work and writing were performed by Dong-mei Wang, Hui Fu, Lin Wang. Bo Feng performed molecular docking and revision of the first draft of paper. Biological Evaluation were performed by Ai-lin Liu and Guan-hua Du. Song Wu supervised the whole project. All authors reviewed and approved the final version.

Conflicts of Interest: The authors declare no conflict of interest.

\section{References}

1. Goedert, M.; Spillantini, M.G. A century of Alzheimer's disease. Science 2006, 314, 777-781. [CrossRef] [PubMed] 
2. Shankar, G.M.; Li, S.; Mehta, T.H.; Garcia-Munoz, A.; Shepardson, N.E.; Smith, I.; Brett, F.M.; Farrell, M.A.; Rowan, M.J.; Lemere, C.A. Amyloid- $\beta$ protein dimers isolated directly from Alzheimer's brains impair synaptic plasticity and memory. Nat. Med. 2008, 14, 837-842. [CrossRef] [PubMed]

3. Grundke-Iqbal, I.; Iqbal, K.; Tung, Y.C.; Quinlan, M.; Wisniewski, H.M.; Binder, L.I. Abnormal phosphorylation of the microtubule-associated protein tauin Alzheimer cytoskeletal pathology. Proc. Natl. Acad. Sci. USA 1986, 83, 4913-4917. [CrossRef] [PubMed]

4. Gella, A.; Durany, N. Oxidative stress in Alzheimer disease. Cell Adhes. Migr. 2009, 3, 88-93. [CrossRef]

5. Rosini, M.; Simoni, E.; Milelli, A.; Minarini, A.; Melchiorre, C. Oxidative Stress in Alzheimer's Disease: Are We Connecting the Dots? Miniperspective. J. Med. Chem. 2014, 57, 2821-2831. [CrossRef] [PubMed]

6. Schelterns, P.; Feldman, H. Treatment of Alzheimer's disease; current status and new perspectives. Lancet Neurol. 2003, 2, 539-547.

7. Smith, D.A. Treatment of Alzheimer's disease in the long-term-care setting. Am. J. Health Syst. Pharm. 2009, 66, 899-907. [CrossRef] [PubMed]

8. Pepeu, G.; Giovannini, M.G. Cholinesterase inhibitors and beyond. Curr. Alzheimer Res. 2009, 6, 86-96. [CrossRef] [PubMed]

9. Grossberg, G.T.; Pejović, V.; Miller, M.L.; Graham, S.M. Memantine therapy of behavioral symptoms in community-dwelling patients with moderate to severe Alzheimer's disease. Dement. Geriat. Cogn. Disord. 2009, 27, 164-172. [CrossRef] [PubMed]

10. Mesulam, M.M.; Guillozet, A.; Shaw, P.; Levey, A.; Duysen, E.G.; Lockridge, O. Acetylcholinesterase knockouts establish central cholinergic pathways and can use butyrylcholinesterase to hydrolyze acetylcholine. Neuroscience 2002, 110, 627-639. [CrossRef]

11. Perry, E.K.; Perry, R.H.; Blessed, G.; Tomlinson, B.E. Changes in brain cholinesterases in senile dementia of Alzheimer type. Neuropathol. Appl. Neurobiol. 1978, 4, 273-277. [CrossRef] [PubMed]

12. Giacobini, E.; Spiegel, R.; Enz, A.; Veroff, A.E.; Cutler, N.R. Inhibition of acetyl-and butyryl-cholinesterase in the cerebrospinal fluid of patients with Alzheimer's disease by rivastigmine: Correlation with cognitive benefit. J. Neurol. Transm. 2002, 109, 1053-1065. [CrossRef] [PubMed]

13. Greig, N.H.; Utsuki, T.; Ingram, D.K.; Wang, Y.; Pepeu, G.; Scali, C.; Yu, Q.S.; Mamczarz, J.; Holloway, H.W.; Giordano, T. Selective butyrylcholinesterase inhibition elevates brain acetylcholine, augments learning and lowers Alzheimer $\beta$-amyloid peptide in rodent. Proc. Natl. Acad. Sci. USA 2005, 102, 17213-17218. [CrossRef] [PubMed]

14. Horton, D.A.; Bourne, G.T.; Smythe, M.L. The combinatorial synthesis of bicyclic privileged structures or privileged substructures. Chem. Rev. 2003, 103, 893-930. [CrossRef] [PubMed]

15. Hajduk, P.J.; Bures, M.; Praestgaard, J.; Fesik, S.W. Privileged molecules for protein binding identified from NMR-based screening. J. Med. Chem. 2000, 43, 3443-3447. [CrossRef] [PubMed]

16. Fang, J.; Yang, R.; Gao, L.; Zhou, D.; Yang, S.; Liu, A.L.; Du, G.H. Predictions of BuChE Inhibitors Using Support Vector Machine and Naive Bayesian Classification Techniques in Drug Discovery. J. Chem. Inf. Model. 2013, 53, 3009-3020. [CrossRef] [PubMed]

17. Ellman, G.L.; Courtney, K.D.; Andres, V., Jr.; Feather-Stone, R.M. A new and rapid colorimetric determination of acetylcholinesterase activity. Biochem. Pharmacol. 1961, 7, 88-95. [CrossRef]

Sample Availability: Samples of the compounds 12-33 are available from the authors.

(C) 2017 by the authors; licensee MDPI, Basel, Switzerland. This article is an open access article distributed under the terms and conditions of the Creative Commons Attribution (CC BY) license (http:/ / creativecommons.org/licenses/by/4.0/). 